\title{
Ueber Röntgenkontrastmittel
}

(Grundsätzliches zur Röntgenologie des Magendarmkanals). Von Dr. W. Bauermeister in Braunschweig.

Im Jahre 1915 ${ }^{1}$ ) hatte ich das Citobaryum als Kontrastmittel in die Röntgenologie eingeführt, ein von E. Merck hergestelltes Baryumpräparat, mit dem man durch einfaches Verrühren mit einer entsprechenden Wassermenge innerhalb weniger Minuten ein allezeit bereites Kontrastmittel herstellen kann, von je nach Bedarf dickbreiiger, sämig-flüssiger bis dünnflüssiger Konsistenz. Mein Bestreben, micli von der Kochküche frei zu machen, hat Nachahmer gefunden. Nachdem vor längerer Zeit empfohlen wurde, als Kontrastmittel einfach in Milch verrührtes Baryum sulfuricum zu verwenden, wird neuerdings ein "Eubaryt" 2 ) genanntes Baryumpräparat angegeben, das ebenfalls nur mit einer entspreclienden Wassermenge zu verrühren ist. $O b$ diese beiden Präparate alle Eigenschaften besitzen, die an ein in jeder Beziehung brauchbares oder gar vollwertiges Kontrastmittel zu stellen sind, erscheint mir nach meinent Versuchen zweifelhaft. Zwischen dem Citobaryum, dem ich diese Eigenschaften unter allen Umständen zuschreibe, und beiden genannten Präparaten besteht zunächst ein fundamentaler Unterschied: während das Citobaryum eine Emulsion darstellt die sich auch bei tagelangem Stehen so gut wie gar nicht abscheidet, sind die beiden anderen Kontrastmittel nur einfache Suspensionen, die in der $\mathrm{Zu}$ sammensetzung von $150 \mathrm{~g}$ Kontrastmittel auf $400 \mathrm{ccm}$ Flüssigkeit innerhalb Minuten schon auszufallen beginnen. [Eine zielbewußte Auffüllurig des Magens erfordert ein Volumen von ca. $500 \mathrm{ccm}$ Kontrastmittel; eine Aufschwemmung von $150 \mathrm{~g}$ Baryum in $200 \mathrm{ccm}$ Flüssig. keit ist dafür praktisch unbrauchbar.] Wenn es nun bei der Röntgenologie des Magendarmkanals darauf ankäme, eine bestimmte Menge Kontraststoff möglichst schnell, ,herunterzustürzen", so könnte man mit jenen beiden genannten Präparaten ausgezeichnet fahren. Aber die eigentliche Röntgenologie des Magendarmkanals, in zwecksinniger Form durchgefülurt, erfordert meines Erachtens ein Kontrastmittel mit Eigenschaften, denen gegenüber die leichte und schnelle Einverleibung eine geringe Rolle spielt. Ja, ich stehe nicht an, in letzterer Möglichkeit unter Umständen einen direkten Mangel zu erblicken. Es ist eine unumstößlich feststehende Tatsache, daß wir bei der Röntgenologie der Speiserölre und des Magens gerade die wichtigsten Zeichen während der Auffüllung und der dabei vor sich gehenden Entfaltung dieser Organe sehen, und, um sie bestimmt als solche Merkmale erkennen zu können, dazu gehört eine gewisse Langsamkeit ihres Auftretens. Zum Beispiel: Ein Schluck dünnflüssiger Kontrastmilch jagt durch die Speiseröhre mit einer Schnelligkeit, daß sie bereits restlos in den Maren verschwunden ist, ehe wir sozusagen zur Besinnung kommen. Benutzen wir dagegen zur Aufweitung der Speiseröhre eine dicksämige, eben trinkbare Citobaryumlösung, so greht die Entfaltung derselhen so langsam vor sich, daß wir die einzelnen Phasen dabei mit Muße feststellen können. Die der Citobaryumasıfschwemmung innewohnende Kohärenz schaltet die einfachen Schwergesetze, nach denen Flüssigkeiten einfach hinabfallen, vollständig aus. Ja, in Verbindung mit der dem Präparat ebenfalls eigenen Eigenschaft der unter Umstănnden sogar zähen Adliärenz an der Umgebung wird aus dem rein passiven Vorgang der Durchströmung einer Flüssigkeit eine aktive Leistung der Oesophagusmuskulatur, von deren Ablauf wir uns auf dem Leuchtschirm ganz in Ruhe überzeugen können. Bei einer sämigen Emulsion finden wir z. B. eine Störung der Passage schon da. wo cine flüssige Darreichungsform glatt durchflieBen würde. Eine steife Emulsion wird zu einer siclitharen dauernden Stase führen, ohne daß wir feste Kontrastmittel (Bissen, Kapseln) zu verabfolgen brauchen, deren Anwendung selbstverständlich in gewissen Fällen zu weiterer Nachprüfung dann noch freisteht.

Das hier entwickelte Prinzip festweicher Kontrastmi'tel ist noch wichtiger für die Röntgenologie des Magens selbst. Der Streit, ob Leuchtschirm, ob Phótographie, ist längst dahin entschieden, daB wir die wichtigsten diagnostischen Zeichen auf dem Leuchtschirun sehen, daß wir hier vor allen Dingen die Hinweise erst finden, ob, wo und wann eine photographische Fixierung von Wert ist. Und vor allen Dingen liefert uns hierbei - ich habe das immer wieder betont - das Studium der sogenannten Entfaltung ${ }^{3}$ ) die wertvollsten Aufschlüsse. Gerade während der Entfa'tung treten nicht selten dirckt pathognomonische Zeichen auf, die bei weiterer Füllung namentlich in dem vollendeten Schattenbilde wieder vollständig untergehen und die wir ebendeshalb gerade in einem bestimmten Stadium der Entfaltung sehen und auf die photographische Platte bringen inüssen. Alles das ist aber auch nur möglich unter

1) D. m. W. 1915 Nr. $26 .-$ - M. m. W. 1920 Nr. 27. - ") Entfaltung, Entleerung, Einfaltung des Magens, Boas, Archiv $24 \mathrm{H}, 5$. 
Anwendung eines Kontrastmittels, unter dem die Entfaltung in regelmäßiger Langsamkeit vor sich geht. Aus diesem Grunde ist eine sämig-trinkbare Citobaryumemulsion allen flüssigen Kontrastmitteln überlegen, und diesem Verfahren verdanke ich beispielsweise, daß ich in den letzten 5 Jahren ca. 100 Fälle von Ulcus penetrans, Ulcus perforans und Sanduhrmagen auf die Platte bekommen habe. Die planmäBige Röntgenuntersıchung des Magens erfordert, daB wir ihn in gewissen Abständen weiter besehen, utn z. B. nun Anhaltspunkte über die Sekretions- und Motilitätsverhältnisse zu gewinnen, und hier gibt die Anwendung der flüssigen Kontrastmittel zu schwersten Bedenken AnlaB. Wenn wir zwanzig Minuten nach CitobaryumgenuB über dem horizontal abschneidenden Kontrastschatten eine Flüssigkeitssäule konstatieren, so handelt es sich bestimmt um einen Magensaftfluß, denn die Citobaryumemulsion ist beständig. Derselbe Befund bei beispielsweise erfolgter Verabreichung des Eubaryttrankes sagt uns dagegen gar nichts, denn analog den Reagenzglasversuchen und wie wiederholte Vergleichsuntersuchungen beim Magen selbst gezeigt haben, sind sowohl die Baryum-Milchaufschwemmung wie der Eubaryttrank Suspensionen, die den eigentlichen Kontraststoff in besagter Zeit wieder ausfallen lassen, ebenfalls mit Bildung einer überstehenden Flüssigkeitsschicht, die in dem Fall natürlich kein abgesonderter Magensaft sein kann.

Für die Beurteilung der Motilität wird die Zeit zugrunde. gelegt, in welcher sich der Magen des Kontrastmittels wieder entledigt. Die Beurteilung muB um so sicherer sein, je breiter das AusmaB ist. Die alte Wismutmahlzeit erfordert 4-6 Stunden zu ihrem Abschub. Die Restbestimmungen in der dritten, vierten, fünften Stunde lieferten ziemlich sichere Grundlagen für die Beurteilung der motorischen Leistung; ebenso der entsprechende Verweilrest des Citobaryum-Kontrastmittels, welches den Magen normaliter in 2-21/9 Stunden verläßt. Die neuen flüssigen Kontrastmittel verlassen aber den Magen bereits wieder in einer so kurzen Zeit (z. B. in 50-80 Minuten), die Zeitzahlen werden so zusammengedrängt, daß eigentlich Minutenbestimmungen Platz greifen müBten, und damit verliert natürlich diese ganze Bestimmungsweise auBerordentlich an Zuverlässigkeit und Bedeutung.

Diese Ausführungen mögen genügen, um den Wert der ${ }^{*}$ enannten Kontrastmittel gegelleinander abzuwägen: sie sind nicht nur pro domo, sondern vielmehr pro re geschrieben, und in dieser Beziehung muB ich die interessierten Kreise immer und immer wieder auf die Wichtigkeit eines genauen Studiums der Entfaltungsvorgänge aufınerksam machen, und iclı stehe nicht an, die Citobaryumemulsion nach wie vor als das Mittel anzusprechen, welches vermöge seiner obengenannten physikalischen Eigenschaften diagnostisch alles aus der Röntgenuntersuchung herausholt, was daraus herauszuholen ist. 